OPEN ACCESS

Edited by:

Jie Xu,

Shanghai Jiao Tong University, China

Reviewed by:

Stefaan Willy Van Gool,

KU Leuven, Belgium

Sheng Wang,

Fudan University, China

*Correspondence:

Frédéric Amant

frederic.amant@uzleuven.be

Massimo Nabissi

massimo.nabissi@unicam.it

tThese authors have contributed equally to this work

Specialty section:

This article was submitted to Pharmacology of Anti-Cancer Drugs,

a section of the journal

Frontiers in Oncology

Received: 11 July 2019 Accepted: 30 September 2019

Published: 15 October 2019

Citation:

Marinelli O, Annibali D, Aguzzi C,

Tuyaerts S, Amant F, Morelli MB, Santoni G, Amantini C, Maggi F and Nabissi M (2019) The Controversial Role of PD-1 and lts Ligands in

Gynecological Malignancies.

Front. Oncol. 9:1073.

doi: 10.3389/fonc.2019.01073

\section{The Controversial Role of PD-1 and Its Ligands in Gynecological Malignancies}

\author{
Oliviero Marinelli $i^{1,2 t}$, Daniela Annibali ${ }^{3+}$, Cristina Aguzzi ${ }^{1}$, Sandra Tuyaerts ${ }^{3}$, \\ Frédéric Amant ${ }^{3,4 *}$, Maria Beatrice Morelli ${ }^{1,2}$, Giorgio Santoni ${ }^{1}$, Consuelo Amantini ${ }^{2}$, \\ Federica Maggi ${ }^{5}$ and Massimo Nabissi ${ }^{1 *}$ \\ ${ }^{1}$ School of Pharmacy, University of Camerino, Camerino, Italy, ${ }^{2}$ School of Bioscience and Veterinary Medicine, University of \\ Camerino, Camerino, Italy, ${ }^{3}$ Gynecological Oncology, Oncology Department, LKI Leuven Cancer Institute KU \\ Leuven-University of Leuven, Leuven, Belgium, ${ }^{4}$ Centre for Gynecologic Oncology Amsterdam (CGOA), Antoni Van \\ Leeuwenhoek-Netherlands Cancer Institute (AvL-NKI), University Medical Center (UMC), Amsterdam, Netherlands, \\ ${ }^{5}$ Department of Molecular Medicine, Sapienza University, Rome, Italy
}

The programmed death-1 (PD-1, CD279) receptor with its ligands, programmed death ligand 1 (PD-L1, CD274, B7-H1), and programmed death ligand 2 (PD-L2, CD273, B7-DC), are the key players of one of the immune checkpoint pathways inhibiting T-cell activation. PD-L1 and PD-L2 are expressed in different cancer cells and their microenvironment, including infiltrating immune cells. However, their prognostic value is still debated and their role in the tumor microenvironment has not been fully elucidated yet. Considering the importance that cancer immunotherapy with anti-PD-1 and anti-PD-L1 antibodies gained in several tumor types, in this review article we aim to discuss the role of the PD-1/PD-L1/PD-L2 axis in gynecological cancers. PD-1 ligands have been detected in ovarian, cervical, vulvar and uterine cancers, and correlation with prognosis seems dependent from their distribution. About PD-L2, very few reports are available so far in gynecological malignancies, and its role is still not completely understood. Clinical trials using anti-PD-1 or anti-PD-L1 antibodies, but not anti-PD-L2, are currently ongoing, in all types of gynecological cancers. They have shown good safety profiles in a certain cohort of patients, but response rates remain low and many aspects remain controversial. In this review, we propose possible solutions to enhance the clinical efficacy of PD-1 axis targeting therapies. Regarding PD-L2, it might be useful to better clarify its role in order to improve the efficiency of immunotherapy in female malignancies.

Keywords: PD-L2, PD-L1, PD-1, ovarian cancer, endometrial cancer, cervical cancer, immunotherapy

\section{INTRODUCTION}

\section{PD-1 and Its Ligands, PD-L1 (B7-H1) and PD-L2 (B7-DC)}

Programmed death-1 (PD-1, CD279) receptor and its ligands, programmed death ligand 1 (PD-L1, CD274, B7-H1) and programmed death ligand 2 (PD-L2, CD273, B7- DC), play crucial roles in one of the immune checkpoint pathways responsible for the inhibition of T-cell activation (1).

$\mathrm{PD}-1$ receptor belongs to the CD28 family and is mainly expressed on the cellular surface of activated $\mathrm{T}$ and $\mathrm{B}$ cells, monocytes, natural killer (NK), and dendritic cells (DCs), with a role in the induction and maintenance of peripheral tolerance and for the maintenance of the stability and 
the integrity of T cells (2-5). PD-1 ligands are glycoproteins, members of the B7 family, with $40 \%$ homology in amino acids sequence, but have quite distinct expression patterns, being expressed by a wide variety of immune and non-immune cells $(1,3,4)$.

PD-L1 is a type I transmembrane glycoprotein with a single $\mathrm{N}$-terminal immunoglobulin variable (IgV)-like domain sharing 21-33\% sequence identity with CTLA-4, CD28, and ICOS, about 20 amino acids that separate the IgV domain from the plasma membrane, a transmembrane domain and a cytoplasmic tail (4). It is constitutively expressed on activated T and B cells, DCs, macrophages, mesenchymal stem cells, and bone marrowderived mast cells $(4,6)$. Additionally, it is expressed on a wide variety of non-hematopoietic cells including the vascular endothelium, fibroblastic reticular cells, keratinocytes, lung, nonparenchymal cells of the liver, mesenchymal stem cells, pancreatic islet cells, astrocytes, and neurons $(4,5,7)$. PD-L1 expression on human $\mathrm{T}$ cells is induced by common $\gamma$ chain cytokines (IL-2, IL-7, and IL-15), whereas PD-L1 expression on B cells is stimulated by IL-21 (4). In cancer cells, PD-L1 expression is regulated by the MAPK and PI3K/AKT pathways, as well as by HIF- $1 \alpha$, STAT- 3 , NF- $\kappa$ B and epigenetic mechanisms via microRNAs (8). PD-L1 also exists in a soluble form (sPD-L1) that originates from the cleavage of membrane-bound PD-L1 by matrix metalloproteinases. Such PD-L1 soluble isoform, mainly produced by myeloid-derived cells, retains the IgV-like domain, necessary for the interaction with PD-1, and it is able to suppress T-cell activation. However, its physiological role is still unknown. Interestingly, sPD-L1 has been found in several human cancer cell lines, including H1299 non-small cell lung cancer cells, U937 lymphoma cells, HO8910 ovarian carcinoma cells, SPCA1 lung adenocarcinoma cells and U251 glioblastoma cells. In addition, high plasma levels of sPD-L1 have been associated with metastasis and poor prognosis in breast cancer and diffuse large B-cell lymphoma (8).

$\mathrm{PD}-\mathrm{L} 2$ is a type I transmembrane protein containing an IgV-like domain and an immunoglobulin constant ( $\operatorname{IgC}$ )-like domain in its extracellular region (9). PD-L2 expression is mainly restricted to antigen-presenting cells (APCs), including macrophages and myeloid DCs $(6,7)$, and non-hematopoietic tissues, such as the lung (10), human umbilical vein endothelial cells, and fibroblasts $(1,5)$. Three isoforms of PD-L2 have been described that might influence the outcome of the immune response (9). The most common splice variant contains all 6 exons. In humans, an alternative variant with a spliced-out exon 3 , resulting in a protein lacks the IgC-like domain and with a shorter-extracellular region has been reported. A third isoform misses the transmembrane domain, because exon 3 is spliced out to an alternative acceptor site within exon 4 , and the protein is secreted as a soluble form. This evidence underscores the importance of post-transcriptional regulation in the expression and function of PD-L2. He et al. suggested that isoforms II and III should be able to interact with PD-1, but further confirmation is needed (9).

Exposure to IL-4, IFN- $\gamma$, IL-2, IL-7, IL-15, IL-21, and toll-like receptor ligands induces PD-L2 upregulation in DCs and macrophages (1). Additionally, IL-4, in the presence of respiratory syncytial virus infection, stimulates PD-L2 expression in alveolar epithelial cells $(1,10)$.

Stimulation by tumor necrosis factor alpha (TNF- $\alpha$ ) and interferon gamma (IFN- $\gamma$ ) enhances the constitutive expression of PD-L2 on endothelial cells from human umbilical vein in vitro (1). The NF-kB and the STAT-6 pathways are two major signaling reported to regulate PD-L2 expression (1).

Different molecular mechanisms dictate PD-Ls binding to PD-1, as demonstrated by the crystallographic structures of the complexes, showing that PD-Ls cross-compete and that the concurrent presence of both ligands might modify the functional outcome of the binding (11). Specifically, PD-L1 binding to PD1 requires complex conformational changes of the ligand, while PD-L2 directly interacts with PD-1, explaining its reported 2 to 6fold higher affinity for the receptor (1). Consequently, when both ligands are expressed at similar levels, PD-L2 would be expected to outcompete PD-L1 for binding to PD-1. However, PD-L2 is generally expressed at lower levels in physiological conditions, such as during maturation of DCs by LPS, when PD-L1 acts as the main ligand of $\mathrm{PD}-1$. A known exception is Th2 responses, where PD-L2 is predominant $(1,11)$.

Regarding the PD-1/PD-L1 and PD-1/PD-L2 pathways involved in T cell immune evasion, different reports have been published, mainly regarding the biochemical signaling regulated by the PD-1/PD-L1. It was reported that the binding of PDL1 to PD-1 may cause $\mathrm{T}$ cell apoptosis, anergy, exhaustion, and interleukin-10 (IL-10) expression, suggesting that PD-L1 can act as a defender for PD-L1 $1^{+}$cancer cells from $\mathrm{CD} 8^{+} \mathrm{T}$ cell-mediated lysis $(12,13)$ (Figure 1).

Regarding the PD-L2/PD-1 signaling pathways, it may not be biologically identical, since Repulsive Guidance Molecule B (RGMb) is also a binding partner for PD-L2 (14). Thus, the PDL2 blockade may evocate different cellular responses, depending on the binding partner interaction, which can lead to potential varied biological outcomes. Up to now, in human anti-tumor immunity, the relationship between PD-1, PD-L1, and PDL2 in their cellular expression profile and regulation, potential interactions and biological is considered not completely defined.

\section{PD-1 Ligands in the Tumor Microenvironment Influence the Anti-tumor Response}

PD-L1 and PD-L2 are expressed in different cancer cells and in their microenvironment $(4,8)$, including infiltrating immune cells $(15,16)$. However, their prognostic value is still debated and the role they might play when expressed in the tumor microenvironment has not be fully elucidated yet (17).

Previous evidence shows that PD-L1 expression by cancer cells correlates with poor prognosis (18), while PD-L1 expression by tumor-infiltrating immune cells is associated with improved overall survival (OS) (16). Furthermore, it seems that PDL1 expressed by APC, rather than cancer cells, is essential for the response to immune checkpoint blockade therapy (19). Specifically, survival analysis showed that the presence of PD-L1 on macrophages had a protective role and enhanced the prognosis of patients with hepatocellular carcinoma. 


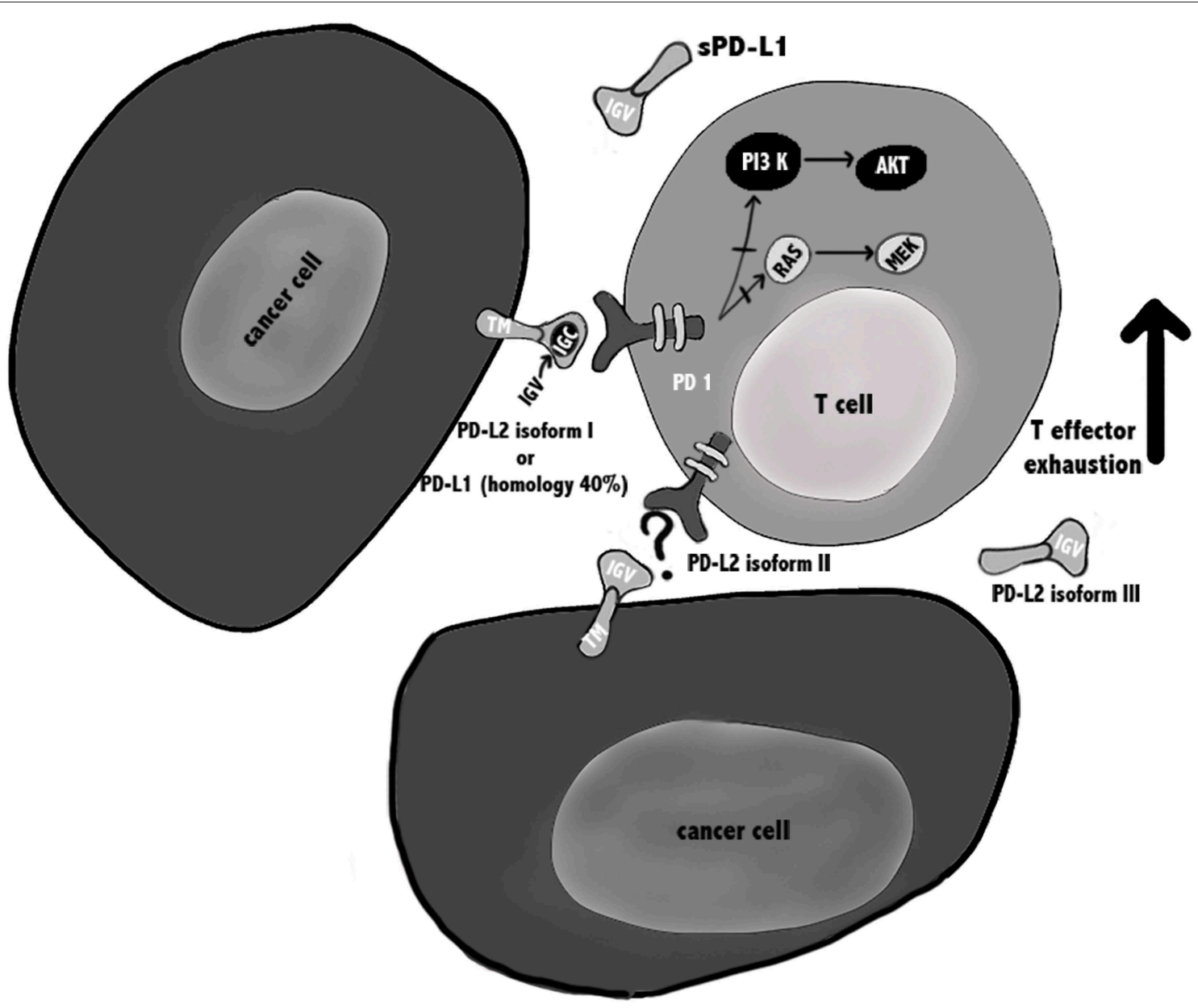

FIGURE 1 | PD-1/PD-Ls pathways in cancer. PD-L1 is a type I transmembrane glycoprotein with a single N-terminal IgV-like domain and exists also in a soluble form SPD-L1 that retains the IgV-like domain. PD-L2 is a type I transmembrane protein containing an IgV-like domain and an IgC-like domain and three isoforms of PD-L2 have been described that might influence the outcome of the immune response. It is suggested that isoforms II and III should be able to interact with PD-1, but further confirmation is needed. During TCR cross-linking, PD-1 by interacting with its ligands, causes inhibition of PI3K/Akt/mTOR and Ras/MAPK/Erk pathways, leading to down-regulation of $T$ cells metabolism, and exhausted $T$ cells.

Macrophages are involved in maintaining an active immune microenvironment, with high numbers of infiltrating $\mathrm{CD}^{+} \mathrm{T}$ cells and high immune-related gene expression levels (15).

Sepesi et al. investigated PD-L1 expression in surgically resected stage I non-small cell lung cancer and, in contrast, demonstrated that lower PD-L1 expression in the tumor, but also in tumor-infiltrating macrophages, was associated with significantly better OS (20).

The existence of conflicting reports about PD-L1 and-2 prognostic value can be generally attributed to technical disparities (e.g., variations in staining protocols across individual laboratories and use of different primary antibody clones to identify PD-Ls in tumor tissue), as well as different clinical features of the analyzed samples (site and size of cancer, treatments, follow-up time, etc.). Moreover, PD-L1 and-2 are dynamic markers that can be up- or downregulated over time, making their evaluation complicated $(17,21)$.
Direct activation of the PD-1 axis by cancer cells leads to a potent inhibitory signal in $\mathrm{T}$ lymphocytes resulting in antitumor immunity impairment and tumor cells ability to escape immunosurveillance $(4,19)$. Specifically, it has been shown that PD-1 activation inhibits glucose consumption, cytokine production, proliferation and survival in T lymphocytes, thus preventing the expression of transcription factors associated with effector $\mathrm{T}$ cell functions, such as GATA-3, T-bet, and Eomesodermin (Eomes) (4). PD-1/PD-Ls binding attenuates TCR-mediated signaling, thus impairing PI3K/Akt and Ras/MEK/Erk pathways, both required for T-cell activation (4).

PD-Ls are expressed in several solid tumors $(8,22)$, and immune checkpoint inhibitors, such as anti-PD-1 and anti-PDL1 antibodies, showed efficacy in cancers with high mutational load, including lung cancer, melanoma, and microsatellite instable (MSI) tumors (23). It was shown that this efficacy is linked to the presence of tumor specific neoantigens that induce a Th1/CTL response that is counterbalanced by overexpression 
of multiple immune checkpoints such as PD-1/PD-L1 (23). In addition, PD-1/PD-L1 axis blockade might activate tumorspecific T lymphocytes to kill tumor cells by inducing TNF- $\alpha$ and IFN- $\gamma(22)$.

For gynaecologic malignancies, the expression of PD-1 ligands has been reported in ovarian $(17,21,22,24-31)$, uterine (5$7,32-38)$, cervical $(23,32,39-50)$, and vulvar $(32,51-54)$ cancers, which we describe in detail in the next section.

\section{PD-1 AND PD-LS EXPRESSION IN ENDOMETRIAL CANCER}

In normal endometrium the role of the immune system is extremely complex, since it must prevent sexually transmitted infections but should also be able to help the growth of an allogenic fetus during pregnancy (23). So far, few reports characterized PD-1 and its ligands' expression in gynecological cancer and data are quite controversial. The expression profile of these immune checkpoints has been analyzed predominantly by immunohistochemistry, in biopsies obtained from both healthy subjects and cancer patients.

\section{PD-1 in Endometrial Cancer}

The $\mathrm{PD}-1$ receptor has been found almost exclusively in immune cells infiltrating the tumor $(32,37,38)$, and not in normal endometrium (5). Additionally, a deep analysis performed on 183 patients showed that high expression of $\mathrm{PD}-1$ within and at the margins of a tumor, with a high $\mathrm{PD}-1 / \mathrm{CD}^{+}$ratio in the center, was associated with favorable OS (35).

Additional reports found a correlation between PD-1 expression in intraepithelial and peritumoural lymphocytes with DNA polymerase $\varepsilon$ (POLE) mutation and MSI status of the patients $(32,37,38)$. Specifically, it has been reported that PD-1 expression in tumor-infiltrating immune cells was more frequently found in moderately, poorly differentiated endometrial cancers, non-endometrioid type II (serous, clear cell, mucinous) endometrial cancers $(5,35,36)$, and POLE and MSI subgroups $(32,37,38)$.

\section{PD-L1 in Endometrial Cancer}

Regarding PD-1 ligands, all data concordantly showed that PD-L1 is expressed in most of the analyzed specimens (5$7,32-35,37)$, predominantly located in the cytoplasm (5-7). Several studies showed that PD-L1 was expressed in a similarly high percentage of samples in both normal endometrium and endometrial tumors (5-7).

PD-L1 expression in cancer cells correlates with postmenopausal status, high histological grade (grade 3), deep myometrial invasion $(\geq 1 / 2)$, lymphovascular invasion, adjuvant therapy, and MSI status (35). High PD-L1 immuno-reactivity on immune cells, and not on tumor cells, is an independent predictor of adverse progression-free survival (PFS) in all patients, including the microsatellite stable (MSS) subgroup (35). In addition, some reports evidenced that PD-L1 expression in intraepithelial immune cells was significantly more frequent in POLE mutant and MSI tumors, compared to MSS tumors (32, 37, 38), while PD-L1 expression in tumor cells did not differ between POLE mutant, MSI and MSS patients (32).
However, data regarding PD-L1 expression in cancer cells are controversial: one study showed that only 1 out of 116 tumors expressed PD-L1 on tumor cells, but this under-estimation could be linked with the use of tissue microarrays, since PD-L1 expression is known to be heterogenous (37).

Another study regarding gynecological samples, in 47 uterine sarcoma samples, found that PD-L1 expression was upregulated in comparison with normal endometrium, suggesting that this protein is a potential target for immunotherapy (7), while Bregar et al., using a smaller number of samples (10 patients), found that PD-L1 is expressed in only $30 \%$ of specimens (34).

\section{PD-L2 in Endometrial Cancer}

For PD-L2 very few data are available so far, and its expression seems to differ from PD-L1, with no significant difference between normal endometrium and tumor (5-7).

High PD-L2 expression was shown in 30\% of primary endometrial carcinoma patients and $16 \%$ of uterine sarcoma patients, demonstrating the potential of PD-L2 blockade in a limited proportion of uterine cancer patients (7). It has been shown that PD-L1 and PD-L2 expression was more frequent in moderately, poorly differentiated, non-endometrioid endometrial cancer and seems to be correlated with POLE and MSI status $(5,33,36)$. Type II endometrial cancer and poorly differentiated histological features are generally associated with worse prognosis and, in addition, $\mathrm{PD}-1$ axis expression suggests that it may cause immunosuppression to favor tumor growth, thus negatively affecting patients' survival (5).

\section{EXPRESSION OF PD-1, PD-L1, AND PD-L2 IN OVARIAN CANCER}

Ovarian cancer is the most lethal disease among gynecological cancers $(17,22,29-31)$ and is known to be an immunogenic tumor.

\section{PD-1 and PD-L1 in Ovarian Cancers}

Some reports showed that PD-L1 expression is found in epithelial ovarian cancers (EOC) $(17,20,21,24-26,30)$, especially in serous ovarian cancers (SOC) $(28,29)$, ovarian clear cell carcinomas (OCCC) and in malignant ascites (31), a sign of peritoneal carcinomatosis derived from ovarian cancer (22).

In a cohort of 122 patients with OCCC, Zhu et al. showed that 55 cases $(44.7 \%)$, classified as having high PD-L1 expression (PD$\mathrm{L} 1^{\text {high }}$ ), were significantly associated with advanced stages (IIIIV) (22). Cases with high PD-L1 and PD-1 expression showed significantly poorer PFS and OS, compared to those with low PD-L1/PD-1 expression (22, 24, 28, 29). In subgroup analysis, $\mathrm{PD}-\mathrm{L} 1^{\text {high }}$ was associated with poorer prognosis compared to $\mathrm{PD}-\mathrm{L} 1^{\text {low }}$ in platinum-resistant and advanced stages (III-IV) patients (22). Drake et al. analyzed 55 ovarian cancer biopsies and showed that PD-1 was detected in $87 \%$ of the tumors in both stroma and epithelium, while PD-L1 was only present in $33 \%$ of patients, exclusively in high-grade tumors (17). Additionally, they found that low density of PD-1 and PDL1 expressing cells in tumor tissue was significantly associated with advanced disease, failing to show any significant association between survival and PD-1 or PD-L1 expression in ovarian 
cancer (17), while patients with recurrent tumors and increased infiltrating $\mathrm{PD}-1^{+}$immune cells had longer OS (21). The correlation of $\mathrm{PD}-1$ and $\mathrm{PD}-\mathrm{L} 1$ expression with high-grade tumors and stage IV International Federation of Gynecology and Obstetrics (FIGO) disease has also been confirmed by other studies $(28,29)$.

Wieser et al. showed that, in a cohort of 158 patients with high-grade serous ovarian cancers, BRCA1/2 mutated tumors were characterized by high PD-1 expression, and that PD-L1 was observed mainly in BRCA1/2 and TP53 mutated cancers (29). Xiao et al. reported that PD-1 is expressed in tumor infiltrating lymphocytes and PD-L1 in tumor cells and in intratumoural immune cells, but there was no significant difference of $\mathrm{PD}-1^{+}$intratumoural immune cells in tumors with different mismatch repair (MMR) status (30). MSI ovarian cancers exhibited a significantly higher number of $\mathrm{PD}-\mathrm{L}^{+}{ }^{+}$intratumoural immune cells compared to MSS ovarian cancers, while PD-L1 expression was not different in tumors, irrespectively from their MMR status (30).

In addition, no significant difference regarding PD-L1 expression in tumor cells and tumor infiltrating lymphocytes, and PD-1 expression in infiltrating lymphocytes, has been found between primary and recurrent disease (21).

\section{PD-L2 in Ovarian Cancers}

So far, only few studies investigated the expression of PDL2 in ovarian cancer. An analysis on 70 patients showed that PD-L2 expression was not related to patient prognosis or other clinical variables, but negatively correlated with the number of $\mathrm{FOXP}^{+} \mathrm{T}$ regulatory cells (Tregs) (24). Imai et al. analyzed the expression of PD-L1 and PD-L2 on tumor cells and APCs in malignant ascites from epithelial ovarian cancer patients (31), and found differential PD-L1 expression in tumor cells between patients with high or low PD-1expressing $\mathrm{CD}^{+} \mathrm{T}$ cells (43.9 and $27.3 \%$, respectively), while no difference in PD-L1 expression was observed between patients with high and low PD-1 expression on $\mathrm{CD}^{+} \mathrm{T}$ cells (34.1 and 27.3\%, respectively). Between 2.3 and $3.2 \%$ of the patients with high or low PD-1 on $\mathrm{CD}^{+} \mathrm{T}$ cells and $\mathrm{CD}^{+} \mathrm{T}$ cells also expressed PD-L2. No correlation was found between $\mathrm{PD}-\mathrm{L} 1 / 2$ expression and clinical variables or outcomes (31).

To support a potential role of PD-1 and PD-L1/ PD-L2 axis as targets in ovarian cancer, it has been reported in syngeneic orthotopic mouse model of epithelial ovarian cancer, that treatment with anti-PD-1 or anti-PD-L1 antibodies resulted in tumor rejection in $75 \%$ of the treated-mice, while mice treated with anti-PD-L2 antibody did not reject tumors (25). These data can be explained considering the selected models that expressed lower levels of PD-L2 than PD-1 and PD-L1. Additionally, PD-1 and PD-L1 blockade significantly increased the $\mathrm{CD}^{+}$to Tregs and $\mathrm{CD}^{+}$to Tregs ratios within the tumor, while, on the contrary, there was no significant change in the $\mathrm{CD}^{+}$or $\mathrm{CD} 4^{+}$ to Tregs ratios (25).

\section{EXPRESSION OF PD-1, PD-L1, AND PD-L2 IN OTHER GYNECOLOGICAL CANCERS}

Cervical cancer is the third most common gynecological malignancy in Europe (23). Little information is available, up to now, regarding the expression of PD-1 ligands (23, 32, 39, 43-47).

A report from Howitt et al. showed that cervical cancer is a potential candidate for clinical trials testing PD-1 blockade $(23,32,39)$. In fact, using FISH analysis on 48 FormalinFixed Paraffin-Embedded (FFPE) tissue specimens of cervical squamous cell carcinoma, they observed co-amplification or co-gain of PD-L1 and PD-L2 in 32 out of 48 cases (67\%). Immunohistochemical staining for PD-L1 revealed high expression in $95 \%$ of the tumors with membranous staining pattern (32).

Persistent infection with human papilloma virus (HPV) is an essential step in the development of most cervical cancers (40). Some studies hypothesized that HPV may activate PD-1/PD-L1 to evade host immune responses, resulting in persistence of the cervical intraepithelial neoplasia (41). The identification of HPV as an etiological factor leads to antigen production and presentation, thereby making cervical cancer immunogenic (42). Recently, the role of the PD-1/PDL1 axis in HPV associated head and neck squamous cell cancer (HPV-HNSCC) creating an "immune-privileged" site for initial viral infection and subsequent adaptive immune resistance suggests a rationale for therapeutic blockade of this pathway in patients with HPV-associated tumors (43). Significant PD-L1 expression in cervical carcinoma has been confirmed in several studies (44-47). As a consequence, this immunogenic disease requires a highly immunosuppressive microenvironment to progress and metastasize $(48,49)$ which has been demonstrated in tumor-positive lymph nodes where high Treg levels, low $\mathrm{CD}^{+} \mathrm{T}$ cell/Treg ratio and high levels of $\mathrm{PD}-\mathrm{L}^{+}$and $\mathrm{HLA}^{+} \mathrm{DR}^{+}$myeloid cells were found (50).

Regarding another gynecological malignancy, vulvar cancer, the clinical relevance of PD-L1 expression has not been completely studied so far (32).

Although rare, incidence rates of vulvar cancer are increasing and, in locally advanced, metastatic or recurrent disease, prognosis is poor and new treatment modalities are needed (51). Screening of 23 vulvar squamous cell carcinomas revealed 6 cases (26\%) with co-amplification of PD-1 ligands, 4 cases (17\%) showed co-gain, 6 cases (26\%) showed polysomy, and 7 cases (30\%) showed disomy. Immunohistochemical staining for PD$\mathrm{L} 1$ across all cases revealed the highest median $\mathrm{PD}-\mathrm{L} 1$ protein expression in cases with co-amplification of PD-L1 and PDL2, and decreasing values with decreasing genetic complexity (32). Previous studies showed that PD-L1 is expressed in the majority of vulvar squamous cell carcinoma samples (51-54), in both cancer cells and peritumoural immune cells (52-54). Additionally, its expression was related with several components of immune system $\left(\mathrm{CD}^{+}, \mathrm{CD}^{+} 0^{+}\right.$, and $\mathrm{CD}^{+} 8^{+}$intra-tumor immunocytes) $(51,54)$, while a significant correlation with immunosuppressive cell populations $\left(\mathrm{FOxP}^{+}\right.$Treg cells) was reported only by Sznurkowski et al. (54). Data analyzing the 
TABLE 1 | Ongoing immunotherapy clinical trials for patients with endometrial cancer.

\begin{tabular}{llll}
\hline ClinicalTrials.gov identifier & Status & Interventions/alone or in combination & Phase \\
\hline NCT02630823 & Active, not recruiting & Pembrolizumab (anti-PD-1) + Paclitaxel/Carboplatin/Radiation (standard of care) \\
NCT02725489 & Active, not recruiting & Durvalumab (anti-PD-L1) & I \\
NCT02728830 & Active, not recruiting & Pembrolizumab (anti-PD-1) & Early I \\
NCT02646748 & Active, not recruiting & Pembrolizumab (anti-PD-1) + itacitinib/NCB050465 & I \\
NCT02914470 & Active, not recruiting & Atezolizumab (anti-PD-L1) + cyclophosphamide/Carboplatin \\
NCT02521844 & Active, not recruiting & Pembrolizumab (anti-PD-1) + ETC-1922159 \\
\hline
\end{tabular}

TABLE 2 | Ongoing immunotherapy clinical trials for patients with ovarian cancer.

\begin{tabular}{|c|c|c|c|}
\hline ClinicalTrials.gov identifier & Status & Interventions (alone or in combination) & Phase \\
\hline NCT02608684 & Active, not recruiting & Pembrolizumab (anti-PD-1) + Gemcitabine/Cisplatin & $\|$ \\
\hline NCT02728830 & Active, not recruiting & Pembrolizumab (anti-PD-1) & Early I \\
\hline NCT03277352 & Active, not recruiting & Pembrolizumab (anti-PD-1) + INCAGN01876/Epacadostat & $1 / 11$ \\
\hline NCT03312114 & Active, not recruiting & Avelumab (anti-PD-L1) & $\|$ \\
\hline NCT02335918 & Completed & Nivolumab (anti-PD-1) + varlilumab & $|/| \mid$ \\
\hline NCT02915523 & Active, not recruiting & Avelumab (anti-PD-L1) + entinostat & $|/| 1$ \\
\hline NCT02452424 & Completed & Pembrolizumab (anti-PD-1) + PLX3397 & $|/| \mid$ \\
\hline NCT02644369 & Active, not recruiting & Pembrolizumab (anti-PD-1) & $\|$ \\
\hline NCT03073525 & Active, not recruiting & Atezolizumab (anti-PD-L1) & $\|$ \\
\hline NCT02764333 & Active, not recruiting & Durvalumab (anti-PD-L1) + TPIV200 & $\|$ \\
\hline NCT02431559 & Active, not recruiting & Durvalumab (anti-PD-L1) + Pegylated Liposomal Doxorubicin & |//I \\
\hline NCT02914470 & Active, not recruiting & Atezolizumab (anti-PD-L1) + carboplatin, cyclophosphamide & 1 \\
\hline NCT02725489 & Active, not recruiting & Durvalumab (anti-PD-L1) & $\|$ \\
\hline NCT01975831 & Active, not recruiting & MEDI4736 (anti-PD-L1) + Tremelimumab & I \\
\hline NCT03038100 & Active, not recruiting & Atezolizumab (anti-PD-L1) + Carboplatin/Atezolizumab/Bevacizumab & III \\
\hline NCT01772004 & Active, not recruiting & Avelumab (anti-PD-L1) & $1 / 11$ \\
\hline NCT03574779 & Active, not recruiting & TSR-042 (anti-PD-1) + Niraparib/Bevacizumab & $\|$ \\
\hline NCT02521844 & Active, not recruiting & Pembrolizumab (anti-PD-1) + ETC-1922159 & I \\
\hline
\end{tabular}

clinical impact of PD-L1 expression in vulvar cancer reveal that it is not clear whether its expression correlates with clinicopathological parameters.

In summary, no significant associations were observed between PD-L1 presence and typical clinicopathological factors (51), except for tumor stage as reported by Sznurkowski et al. (54), and PD-L1 expression occurs more often in high risk HPVnegative samples (51). Regarding survival analysis, it is reported that PD-L1 expression did not influence the OS $(51,53)$, but patients with primary tumors positive for immune cells-PD-L1 expression had improved OS compared to negative ones (54).

The presence of PD-L1 also seems to be an independent prognostic factor for recurrence free survival (51).

\section{ONGOING IMMUNOTHERAPY CLINICAL TRIALS IN GYNECOLOGICAL MALIGNANCIES}

Several clinical trials are ongoing at the moment, according to the ClinicalTrials.gov database [accessed July 06, 2019], testing anti-PD-1/PD-L1 blockade alone or in combination in patients with endometrial, cervical, vulvar and ovarian cancer, while there are no ongoing clinical trials using anti-PD-L2 (Tables 1-3).

Clinical trials data were collected from ClinicalTrials.gov database, selecting only completed trials or in "Active, not recruiting" status. 
TABLE 3 | Ongoing immunotherapy clinical trials for patients with cervical cancer.

\begin{tabular}{|c|c|c|c|}
\hline ClinicalTrials.gov Identifier & Status & Interventions & phase \\
\hline NCT01975831 & Active, not recruiting & MEDI4736 (anti-PD-L1) + Tremelimumab & 1 \\
\hline NCT02914470 & Active, not recruiting & Atezolizumab (anti-PD-L1) + Carboplatin/Cyclophosphamide & 1 \\
\hline NCT02725489 & Active, not recruiting & Durvalumab (anti-PD-L1) & $\|$ \\
\hline NCT02921269 & Active, not recruiting & Atezolizumab (anti-PD-L1) + Bevacizumab & $\|$ \\
\hline NCT02257528 & Active, not recruiting & Nivolumab (anti-PD-1) & $\|$ \\
\hline
\end{tabular}

\section{Endometrial Cancer}

Regarding endometrial cancer, 6 clinical trials are ongoing (Table 1). Most of them are Phase I clinical trials and preliminary results, reported by the American Society of Clinical Oncology (asco.org), showed that atezolizumab (anti-PD-L1), and pembrolizumab (anti-PD-1) might be promising agents for endometrial cancer treatment.

Most relevant results showed that in a phase I study, 15 patients eligible based on PD-L1 status ( $>5 \%$ of positivity in tumor-infiltrating immune cells) were treated with atezolizumab and evaluated for safety and efficacy. Results showed that atezolizumab had a favorable safety profile and 13\% (2/15) of patients showed a reduction in tumor size. A trend for higher PFS and OS has been observed in patients with high levels of tumor-infiltrating immune cells. Clinical benefit appeared to increase with higher PD-L1 expression, suggesting a link between PD-L1 status and response to atezolizumab. In addition, hypermutation, and/or high immune infiltration may be linked to response to PD-L1 blockade (Clinical trial information: NCT01375842) (55).

In a different phase I clinical trial, pembrolizumab was administered in 24 patients with endometrial carcinoma (excluding sarcomas), failure of prior systemic therapy, and PD-L1 expression in $\geq 1 \%$ of tumor or stromal cells. A reduction in tumor size was confirmed in $13.0 \%$ of the patients, while 3 patients achieved stable disease. PFS and OS rates were 19.0 and $68.8 \%$, respectively. In conclusion, Pembrolizumab demonstrated an acceptable safety profile and anti-tumor activity (Clinical trial information: NCT02054806) (56).

\section{Ovarian Cancer}

For ovarian cancer 22 clinical trials are ongoing, 2 of which are completed (Table 2). Some of the early-phase clinical trials of anti-PD-1 or anti-PD-L1 antibodies have shown good safety profiles and durable anti-tumor response in certain patient population(s). However, their response rates remain between 10 and $15 \%(31,57)$. Available interim reports from some of the trials show promising objective response rates (ORR) for the treatment of ovarian cancer with nivolumab (anti-PD-1) (ORR of 15\%, $n=20$ patients), pembrolizumab (ORR $11.5 \%, n=49$ ), or avelumab (anti-PD-L1) (ORR 10\%, $n=124)(17,58,59)$. Preliminary data presented at the annual ASCO meeting in 2016 of a phase I trial evaluating durvalumab (anti-PD-L1) in combination with olaparib (PARP inhibitor), showed a disease control rate (DCR) of $67 \%$ for the doublet olaparib - durvalumab in a cohort including BRCA wild type triple negative breast cancer and EOC cases (23).

In the KEYNOTE-28 trial, which explored the activity of pembrolizumab in several solid tumors, outcome of ovarian cancer was ORR of $11.5 \%$, and only $23.1 \%$ showed tumor shrinkage from baseline (57).

\section{Cervical Cancer}

For cervical cancer, 6 clinical trials are ongoing (Table 3). Most relevant findings showed that in a phase Ib study with 24 patients affected by advanced cervical squamous cell cancer and PD-L1 expression in $\geq 1 \%$ of tumor or stromal cells, pembrolizumab was well-tolerated and showed promising anti-tumor activity (Clinical trial information: NCT02054806) (60), while its clinical benefit was investigated in the phase 2 KEYNOTE-158 trial. Pembrolizumab administration has been also investigated in a single cohort trial enrolling 98 patients with recurrent or metastatic cervical cancer, expressing PD-L1 with a positive ratio of the number of all PD-L1expressing cells (tumor cells, lymphocytes, macrophages) to the number of all tumor cells, or a Combined Positive Score (CPS) $\geq 1$. The ORR in 77 patients was $14.3 \%$ (95\% CI: 7.4, 24.1 ), including $2.6 \%$ complete responses and $11.7 \%$ partial responses. No responses were observed in patients with tumors negative for PD-L1 expression (CPS <1). Serious adverse reactions occurred in $39 \%$ of patients (Clinical trial information: NCT02628067) (61).

On June 12th 2018, pembrolizumab was approved by Food and Drug Administration (FDA), for treatment of patients with recurrent or metastatic cervical cancer, expressing PD-L1 (CPS $\geq 1$ ) as determined by an FDAapproved test, with disease progression on or after chemotherapy ${ }^{1}$.

In conclusion, since in all gynecological cancers ORR is around $10-15 \%$, this emphasizes the need for combination treatments to improve efficacy of immune checkpoint (Figure 2).

\footnotetext{
${ }^{1}$ Merck \& Co. Press Release Details. https://investors.merck.com/news/pressrelease-details/2018/FDA-Approves-Mercks-KEYTRUDA-pembrolizumab-forPreviously-Treated-Patients-with-Recurrent-or-Metastatic-Cervical-CancerWhose-Tumors-Express-PD-L1-CPS-Greater-Than-or-Equal-to-1/default.aspx
} 


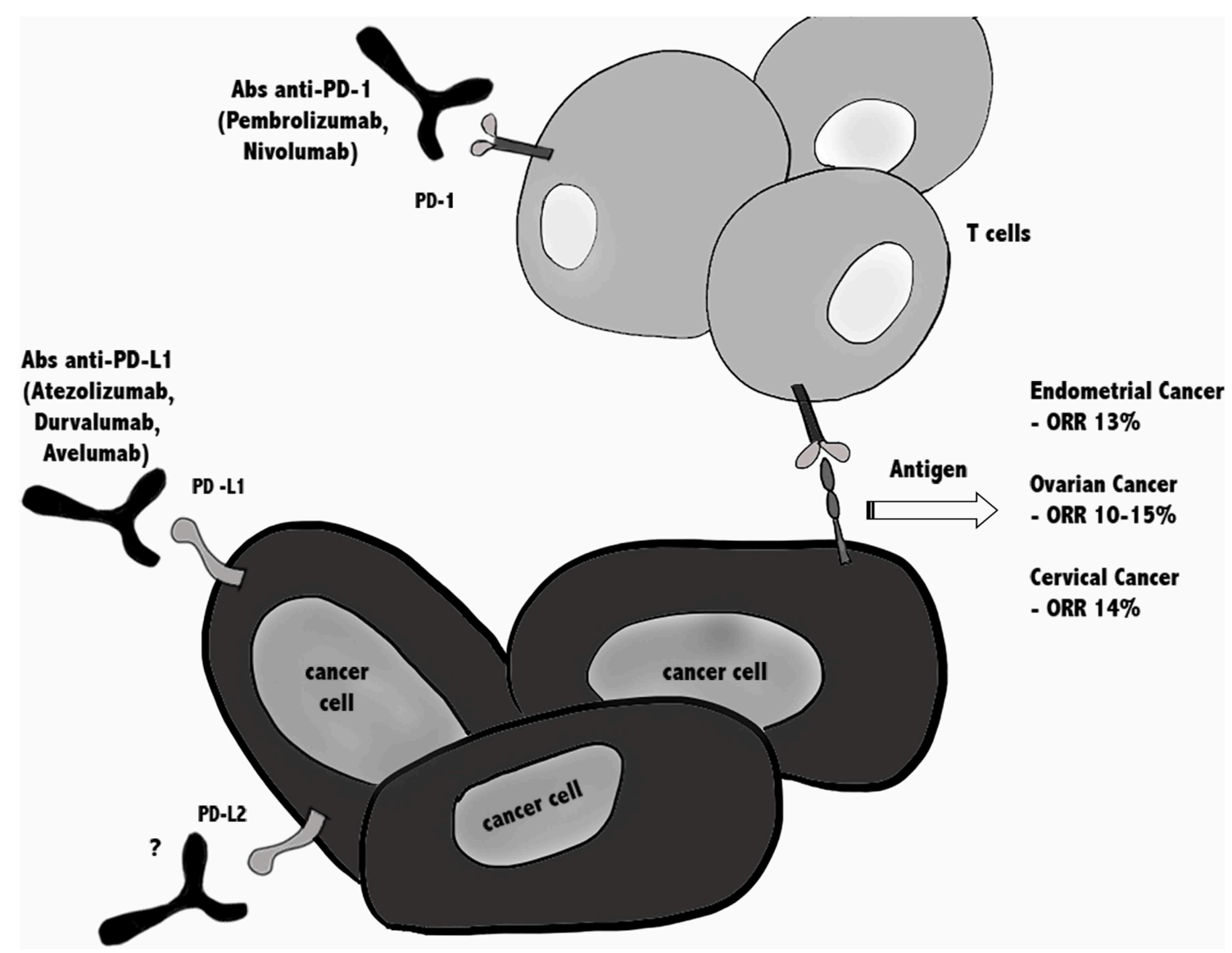

FIGURE 2 | Immunotherapy against PD-1/PD-Ls in gynecological cancers. Blocking the PD-1/PD-L1 immune checkpoint pathway by anti-PD-1 or anti-PD-L1 antibodies suppresses cancer cell survival and enhances the antitumor responses of $\mathrm{T}$ cells, leading to tumor regression and rejection. Actually, several clinical trials are ongoing testing anti-PD-1/PD-L1 blockade alone or in combination, in patients with endometrial, cervical, vulvar, and ovarian cancer, while there are no ongoing clinical trials using anti- PD-L2. In all gynecological cancers ORR is around 10-15\%, argues for combinatorial treatments are taken in consideration.

\section{FUTURE DIRECTIONS FOR IMMUNE CHECKPOINT INHIBITORS (ICIS) COMBINATION THERAPIES}

Albeit ICIs therapies have been shown to induce durable responses and long-term remission in several cancer types, many patients fail to respond, develop resistance over the time or show immune-related adverse effects (62-65). The unresponsiveness or the toxicity of ICIs represents a strong rationale for the combination of ICIs with other treatments to increase the response rate of non-immunological tumors. For example, therapeutic approaches that induce the release and presentation of tumor antigens could be able to foster a de novo anti-tumor $\mathrm{T}$ cell response. In this regard, candidates for a combination therapy with ICIs could be cancer vaccines, oncolytic viruses, radiation, or low-dose chemotherapy (66).

Another potential combination approach with ICIs could be with bispecific antibodies, which recruit patient's T cells or NK cells against cancer cells expressing tumor-associated antigens. An example came from hematologic malignancies, wherein a bispecific antibody targeting both CD3 and CD123 $(67,68)$ was used but showed benefit in only a small fraction of patients. A major mechanism limiting the therapeutic efficacy was $\mathrm{T}$ cell anergy and exhaustion driven by ICIs pathways (mainly PDL1/PD-1) (69). Inspired by this inhibitory role of ICIs pathway, combining ICIs with bispecific antibodies showed enhanced $\mathrm{T}$ cell proliferation and IFN- $\gamma$ production (70).

One more possibility to improve ICI efficacy might be combination with cytokine therapy. The cytokine IL-2 has been approved for the treatment of metastatic renal cell carcinoma and advanced melanoma but is accompanied by severe side effects (71). However, modified IL-2 formulations such as bempegaldesleukin (NKTR-214) have an improved safety profile and have shown capabilities of enhancing the proliferation and activation of $\mathrm{CD}^{+} \mathrm{T}$ cells and NK cells without increasing the number of Tregs (72). Recently, the PIVOT-02 trial (combination of NKTR-214 and nivolumab) has shown that this combination is safe and efficacious (ORR 48\% in 23 patients) in metastatic urothelial carcinoma (73). 
In addition, a recent study has demonstrated that DC-derived IL-12 is necessary for successful anti-PD-1 cancer therapy, suggesting that IL-12 and ICIs could be rationally combined (74).

Finally, there is strong rationale to combine anti-angiogenic therapies with ICI's, since anti-angiogenic therapies induce a normalization of the tumor vasculature, which leads to enhanced infiltration of T lymphocytes in the tumor.

\section{CONCLUSION}

Cancer immunotherapy is emerging as a promising component for cancer therapy. The most promising immunotherapy that showed good results involves antibodies targeting inhibitory immune checkpoint molecules (75).

Results obtained for patients with non-small cell lung cancer, renal cancer, and melanoma are evident and encouraging. However, in gynecological malignancies many aspects remain controversial in preclinical and clinical studies (23). Uncertain is the selection of patients because objective response rates remain low and retrospective analysis on biopsies showed opposing results for OS and PFS in patients with similar pattern of expression of $\mathrm{PD}-1$ and its ligands $(15,17,20-22,24,28,29,32,34)$.

\section{REFERENCES}

1. Rozali EN, Hato SV, Robinson BW, Lake RA, Lesterhuis WJ. Programmed death ligand 2 in cancer-induced immune suppression. Clin Dev Immunol. (2012) 2012:656340. doi: 10.1155/2012/656340

2. Yang S, Zhang Q, Liu S, Wang AR, You Z. PD-1, PD-L1 and PD-L2 expression in mouse prostate cancer. Am J Clin Exp Urol. (2016) 4:1-8.

3. Ohigashi Y, Sho M, Yamada Y, Tsurui Y, Hamada K, Ikeda N, et al. Clinical significance of programmed death-1 ligand-1 and programmed death-1 ligand-2 expression in human esophageal cancer. Clin Cancer Res. (2005) 11:2947-53. doi: 10.1158/1078-0432.CCR-04-1469

4. Bardhan K, Anagnostou T, Boussiotis VA. The PD1:PD-L1/2 pathway from discovery to clinical implementation. Front Immunol. (2016) 7:550. doi: 10.3389/fimmu.2016.00550

5. Mo Z, Liu J, Zhang Q, Chen Z, Mei J, Liu L, et al. Expression of PD-1, PDL1 and PD-L2 is associated with differentiation status and histological type of endometrial cancer. Oncol Lett. (2016) 12:944-50. doi: 10.3892/ol.2016.4744

6. Liu J, Liu Y, Wang W, Wang C, Che Y. Expression of immune checkpoint molecules in endometrial carcinoma. Exp Ther Med. (2015) 10:1947-52. doi: 10.3892 /etm.2015.2714

7. Vanderstraeten A, Luyten C, Verbist G, Tuyaerts S, Amant F. Mapping the immunosuppressive environment in uterine tumors: implications for immunotherapy. Cancer Immunol Immunother. (2014) 63:545-57. doi: 10.1007/s00262-014-1537-8

8. Chen J, Jiang CC, Jin L, Zhang XD. Regulation of PD-L1: a novel role of pro-survival signalling in cancer. Ann Oncol. (2016) 27:409-16. doi: 10.1093/annonc/mdv615

9. He XH, Liu Y, Xu LH, Zeng YY. Cloning and identification of two novel splice variants of human PD-L2. Acta Biochim Biophys Sin. (2004) 36:284-9. doi: $10.1093 /$ abbs/36.4.284

10. Dong Y, Sun Q, Zhang X. PD-1 and its ligands are important immune checkpoints in cancer. Oncotarget. (2017) 8:2171-86. doi: 10.18632/oncotarget.13895

11. Ghiotto M, Gauthier L, Serriari N, Pastor S, Truneh A, Nunès JA, et al. PD-L1 and PD-L2 differ in their molecular mechanisms of interaction with PD-1. Int Immunol. (2010) 22:651-60. doi: 10.1093/intimm/dxq049
Regarding the second ligand PD-L2, it is needed to better clarify its role inside tumor microenvironment, together with the evaluation of other biological markers, in order to improve the efficiency of immunotherapy malignancies of the female genital tract.

\section{AUTHOR CONTRIBUTIONS}

OM, DA, CA, MN, FA, and ST wrote the paper. MM, GS, CA, and FM have revised the clinical trials and the paper.

\section{FUNDING}

This work was supported by grants from Fondazione Umberto Veronesi (Post-doctoral Fellowship 2018, 2019 to MM) and UNICAM School Advanced Studies in Life and Health Sciences.

\section{ACKNOWLEDGMENTS}

Thanks to Dr. Dario Conti for his support on endometrial cancer research in UNICAM. FA was a senior researcher for Research Foundation-Flanders (FWO). ST was financially supported by the Anticancer Fund (www.anticancerfund.org) and by the associated Verelst Uterine Cancer Fund Leuven.

12. Zou W, Chen L. Inhibitory B7-family molecules in the tumour microenvironment. Nat Rev Immunol. (2008) 8:467-77. doi: 10.1038/nri2326

13. Chen L, Han X. Anti-PD-1/PD-L1 therapy of human cancer: past, present, and future. J Clin Invest. (2015) 125:3384-91. doi: 10.1172/JCI80011

14. Xiao Y, Yu S, Zhu B, Bedoret D, Bu X, Francisco LM, et al. RGMb is a novel binding partner for PD-L2 and its engagement with PDL2 promotes respiratory tolerance. J Exp Med. (2014) 211:943-59. doi: $10.1084 /$ jem. 20130790

15. Liu CQ, Xu J, Zhou ZG, Jin LL, Yu XJ, Xiao G, et al. Expression patterns of programmed death ligand 1 correlate with different microenvironments and patient prognosis in hepatocellular carcinoma. Br J Cancer. (2018) 119:80-8. doi: 10.1038/s41416-018-0144-4

16. Birtalan E, Danos K, Gurbi B, Brauswetter D, Halasz J, Kalocsane Piurko $\mathrm{V}$, et al. Expression of PD-L1 on immune cells shows better prognosis in laryngeal, oropharygeal, and hypopharyngeal cancer. Appl Immunohistochem Mo Morphol. (2018) 26:e79-85. doi: 10.1097/PAI.00000000000 00590

17. Drakes ML, Mehrotra S, Aldulescu M, Potkul RK, Liu Y, Grisoli A, et al. Stratification of ovarian tumor pathology by expression of programmed cell death-1 (PD-1) and PD-ligand- 1 (PD-L1) in ovarian cancer. J Ovarian Res. (2018) 11:43. doi: 10.1186/s13048-018-0414-z

18. Pulko V, Harris KJ, Liu X, Gibbons RM, Harrington SM, Krco CJ, et al. B7-h1 expressed by activated CD8 T cells is essential for their survival. J Immunol. (2011) 187:5606-14. doi: 10.4049/jimmunol. 1003976

19. Tang H, Liang Y, Anders RA, Taube JM, Qiu X, Mulgaonkar A, et al. PD-L1 on host cells is essential for PD-L1 blockade-mediated tumor regression. J Clin Invest. (2018) 128:580-8. doi: 10.1172/JCI96061

20. Sepesi B, Cuentas EP, Canales JR, Behrens C, Correa AM, Vaporciyan A, et al. Programmed death cell ligand 1 (PD-L1) is associated with survival in stage I non-small cell lung cancer. Semin Thorac Cardiovasc Surg. (2017) 29:408-15. doi: 10.1053/j.semtcvs.2017.05.008

21. Ojalvo LS, Thompson ED, Wang TL, Meeker AK, Shih IM, Fader AN, et al. Tumor-associated macrophages and the tumor immune microenvironment of primary and recurrent epithelial ovarian cancer. Hum Pathol. (2018) 74:135-47. doi: 10.1016/j.humpath.2017.12.010 
22. Zhu J, Wen H, Bi R, Wu Y, Wu X. Prognostic value of programmed deathligand 1 (PD-L1) expression in ovarian clear cell carcinoma. J Gynecol Oncol. (2017) 28:e77. doi: 10.3802/jgo.2017.28.e77

23. Ventriglia J, Paciolla I, Pisano C, Cecere SC, Di Napoli M, Tambaro R, et al. Immunotherapy in ovarian, endometrial and cervical cancer: state of the art and future perspectives. Cancer Treat Rev. (2017) 59:109-16. doi: 10.1016/j.ctrv.2017.07.008

24. Hamanishi J, Mandai M, Abiko K, Matsumura N, Baba T, Yoshioka Y, et al. The comprehensive assessment of local immune status of ovarian cancer by the clustering of multiple immune factors. Clin Immunol. (2011) 141:338-47. doi: 10.1016/j.clim.2011.08.013

25. Duraiswamy J, Freeman GJ, Coukos G. Therapeutic PD-1 pathway blockade augments with other modalities of immunotherapy T-cell function to prevent immune decline in ovarian cancer. Cancer Res. (2013) 73:6900-12. doi: 10.1158/0008-5472.CAN-13-1550

26. Gatalica Z, Snyder C, Maney T, Ghazalpour A, Holterman DA, Xiao N, et al. Programmed cell death 1 (PD-1) and its ligand (PD-L1) in common cancers and their correlation with molecular cancer type. Cancer Epidemiol Biomarkers Prev. (2014) 23:2965-70. doi: 10.1158/1055-9965.EPI-14-0654

27. Turner TB, Buchsbaum DJ, Straughn JM Jr, Randall TD, Arend RC. Ovarian cancer and the immune system - the role of targeted therapies. Gynecol Oncol. (2016) 142:349-56. doi: 10.1016/j.ygyno.2016.05.007

28. Wang Q, Lou W, Di W, Wu X. Prognostic value of tumor PD-L1 expression combined with $\mathrm{CD} 8(+)$ tumor infiltrating lymphocytes in high grade serous ovarian cancer. Int Immunopharmacol. (2017) 52:7-14. doi: 10.1016/j.intimp.2017.08.017

29. Wieser V, Gaugg I, Fleischer M, Shivalingaiah G, Wenzel S, Sprung $\mathrm{S}$, et al. BRCA1/2 and TP53 mutation status associates with PD-1 and PD-L1 expression in ovarian cancer. Oncotarget. (2018) 9:17501-11. doi: 10.18632 /oncotarget. 24770

30. Xiao X, Dong D, He W, Song L, Wang Q, Yue J, et al. Mismatch repair deficiency is associated with MSI phenotype, increased tumor-infiltrating lymphocytes and PD-L1 expression in immune cells in ovarian cancer. Gynecol Oncol. (2018) 149:146-54. doi: 10.1016/j.ygyno.2018.02.009

31. Imai Y, Hasegawa K, Matsushita H, Fujieda N, Sato S, Miyagi E, et al. Expression of multiple immune checkpoint molecules on $\mathrm{T}$ cells in malignant ascites from epithelial ovarian carcinoma. Oncol Lett. (2018) 15:6457-68. doi: 10.3892/ol.2018.8101

32. Howitt BE, Sun HH, Roemer MG, Kelley A, Chapuy B, Aviki E, et al. Genetic basis for PD-L1 expression in squamous cell carcinomas of the cervix and vulva. JAMA Oncol. (2016) 2:518-22. doi: 10.1001/jamaoncol.2015.6326

33. Sloan EA, Ring KL, Willis BC, Modesitt SC, Mills AM. PD-L1 expression in mismatch repair-deficient endometrial carcinomas, including lynch syndrome-associated and MLH1 promoter hypermethylated tumors. Am J Surg Pathol. (2017) 41:326-33. doi: 10.1097/PAS.0000000000000783

34. Bregar A, Deshpande A, Grange C, Zi T, Stall J, Hirsch H, et al. Characterization of immune regulatory molecules B7-H4 and PD-L1 in low and high grade endometrial tumors. Gynecol Oncol. (2017) 145:446-52. doi: 10.1016/j.ygyno.2017.03.006

35. Kim J, Kim S, Lee HS, Yang W, Cho H, Chay DB, et al. Prognostic implication of programmed cell death 1 protein and its ligand expressions in endometrial cancer. Gynecol Oncol. (2018) 149:381-7. doi: 10.1016/j.ygyno.2018.02.013

36. Kharma B, Baba T, Matsumura N, Kang HS, Hamanishi J, Murakami R, et al. STAT1 drives tumor progression in serous papillary endometrial cancer. Cancer Res. (2014) 74:6519-30. doi: 10.1158/0008-5472.CAN-14-0847

37. Eggink FA, Van Gool IC, Leary A, Pollock PM, Crosbie EJ, Mileshkin L, et al. Immunological profiling of molecularly classified high-risk endometrial cancers identifies POLE-mutant and microsatellite unstable carcinomas as candidates for checkpoint inhibition. Oncoimmunology. (2016) 6:e1264565. doi: 10.1080/2162402X.2016.1264565

38. Yamashita H, Nakayama K, Ishikawa M, Nakamura K, Ishibashi T, Sanuki K, et al. Microsatellite instability is a biomarker for immune checkpoint inhibitors in endometrial cancer. Oncotarget. (2017) 9:5652-64. doi: 10.18632/oncotarget.23790

39. Cancer Genome Atlas Research Network, Albert Einstein College of Medicine, Analytical Biological Services, Barretos Cancer Hospital, Baylor College of Medicine, Beckman Research Institute of City of Hope, et al. Integrated genomic and molecular characterization of cervical cancer. Nature. (2017) 543:378-84. doi: 10.1038/nature21386

40. zur Hausen, H. Papillomaviruses in the causation of human cancers - a brief historical account. Virology. (2009) 384:260-5. doi: 10.1016/j.virol.2008.11.046

41. Zhang $H$, Zhang $T$, You Z, Zhang Y. Positive surgical margin, HPV persistence, and expression of both TPX2 and PD-L1 are associated with persistence/recurrence of cervical intraepithelial neoplasia after cervical conization. PLOS ONE. (2015) 10:e0142868. doi: 10.1371/journal.pone.0142868

42. Alexandrov LB, Nik-Zainal S, Wedge DC, Aparicio SA, Behjati S, Biankin $\mathrm{AV}$, et al. Signatures of mutational processes in human cancer. Nature. (2013) 500:415-21. doi: 10.1038/nature12477

43. Lyford-Pike S, Peng S, Young GD, Taube JM, Westra WH, Akpeng B, et al. Evidence for a role of the PD-1:PD-L1 pathway in immune resistance of HPV-associated head and neck squamous cell carcinoma. Cancer Res. (2013) 73:1733-41. doi: 10.1158/0008-5472.CAN-12-2384

44. Yang W, Lu YP, Yang YZ, Kang JR, Jin YD, Wang HW. Expressions of programmed death (PD)-1 and PD-1 ligand (PD-L1) in cervical intraepithelial neoplasia and cervical squamous cell carcinomas are of prognostic value and associated with human papillomavirus status. J Obstet Gynaecol Res. (2017) 43:1602-12. doi: 10.1111/jog.13411

45. Reddy OL, Shintaku PI, Moatamed NA. Programmed death-ligand 1 (PD-L1) is expressed in a significant number of the uterine cervical carcinomas. Diagn Pathol. (2017) 12:45. doi: 10.1186/s13000-017-0631-6

46. Mezache L, Paniccia B, Nyinawabera A, Nuovo GJ. Enhanced expression of PD L1 in cervical intraepithelial neoplasia and cervical cancers. Mod Pathol. (2015) 28:1594-602. doi: 10.1038/modpathol.2015.108

47. Heeren AM, Punt S, Bleeker MC, Gaarenstroom KN, van der Velden J, Kenter GG, et al. Prognostic effect of different PD-L1 expression patterns in squamous cell carcinoma and adenocarcinoma of the cervix. Mod Pathol. (2016) 29:753-63. doi: 10.1038/modpathol.2016.64

48. Piersma SJ. Immunosuppressive tumor microenvironment in cervical cancer patients. Cancer Microenviron. (2011) 4:361-75. doi: 10.1007/s12307-011-0066-7

49. Pfaendler KS, Tewari KS. Changing paradigms in the systemic treatment of advanced cervical cancer. Am J Obstet Gynecol. (2016) 214:22-30. doi: 10.1016/j.ajog.2015.07.022

50. Heeren AM, de Boer E, Bleeker MC, Musters RJ, Buist MR, Kenter GG, et al. Nodal metastasis in cervical cancer occurs in clearly delineated fields of immune suppression in the pelvic lymph catchment area. Oncotarget. (2015) 6:32484-93. doi: 10.18632/oncotarget.5398

51. Hecking T, Thiesler T, Schiller C, Lunkenheimer JM, Ayub TH, Rohr A, et al. Tumoral PD-L1 expression defines a subgroup of poor-prognosis vulvar carcinomas with non-viral etiology. Oncotarget. (2017) 8:92890-903. doi: 10.18632/oncotarget.21641

52. Chinn Z, Stoler MH, Mills AM. PD-L1 and IDO expression in cervical and vulvar invasive and intraepithelial squamous neoplasias: implications for combination immunotherapy. Histopathology. (2019) 74:256-68. doi: $10.1111 /$ his. 13723

53. Thangarajah F, Morgenstern B, Pahmeyer C, Schiffmann LM, Puppe J, Mallmann P, et al. Clinical impact of PD-L1 and PD-1 expression in squamous cell cancer of the vulva. J Cancer Res Clin Oncol. (2019) 145:1651-60. doi: 10.1007/s00432-019-02915-1

54. Sznurkowski JJ, Zawrocki A, Sznurkowska K, Peksa R, Biernat W. PDL1 expression on immune cells is a favorable prognostic factor for vulvar squamous cell carcinoma patients. Oncotarget. (2017) 8:89903-12. doi: 10.18632/oncotarget.20911

55. Fleming GF, Emens LA, Eder JP, Hamilton EP, Liu JF, Liu B, et al. Clinical activity, safety and biomarker results from a phase Ia study of atezolizumab (atezo) in advanced/recurrent endometrial cancer (rEC). J Clin Oncol. (2017) 35(Suppl.):5585-5585. doi: 10.1200/JCO.2017.35.15_suppl.5585

56. Ott PA, Bang YJ, Berton-Rigaud D, Elez E, Pishvaian MJ, Rugo HS, et al. Safety and antitumor activity of pembrolizumab in advanced programmed death ligand 1-positive endometrial cancer: results from the KEYNOTE028 study. J Clin Oncol. (2017) 35:2535-41. doi: 10.1200/JCO.2017. 72.5952 
57. Dai Y, Sun C, Feng Y, Jia Q, Zhu B. Potent immunogenicity in BRCA1-mutated patients with high-grade serous ovarian carcinoma. J Cell Mol Med. (2018) 22:3979-86. doi: $10.1111 / \mathrm{jcmm} .13678$

58. Iwai Y, Hamanishi J, Chamoto K, Honjo T. Cancer immunotherapies targeting the PD-1 signaling pathway. J Biomed Sci. (2017) 24:26. doi: 10.1186/s12929-017-0329-9

59. Hamanishi J, Mandai M, Ikeda T, Minami M, Kawaguchi A, Murayama T, et al. Safety and antitumor activity of anti-PD-1 antibody, nivolumab, in patients with platinum-resistant ovarian cancer. J Clin Oncol. (2015) 33:4015-22. doi: 10.1200/JCO.2015.62.3397

60. Frenel JS, Le Tourneau C, O'Neil B, Ott PA, Piha-Paul SA, Gomez-Roca C, et al. Safety and efficacy of pembrolizumab in advanced, programmed death ligand 1-positive cervical cancer: results from the phase Ib KEYNOTE-028 trial. J Clin Oncol. (2017) 35:4035-41. doi: 10.1200/JCO.2017.74.5471

61. Chung HC, Schellens JHM, Delord JP, Perets R, Italiano A, Shapira-Frommer $R$, et al. Pembrolizumab treatment of advanced cervical cancer: updated results from the phase 2 KEYNOTE-158 study. J Clin Oncol. (2018) 36(Suppl. 15):5522. doi: 10.1200/JCO.2018.36.15_suppl.5522

62. Callahan MK, Wolchok JD. At the bedside: CTLA-4- and PD-1-blocking antibodies in cancer immunotherapy. J Leukoc Biol. (2013) 94:41-53. doi: $10.1189 / \mathrm{jlb} .1212631$

63. Zitvogel L, Kroemer G. Targeting PD-1/PD-L1 interactions for cancer immunotherapy. Oncoimmunology. (2012) 1:1223-5. doi: 10.4161/onci.21335

64. O'Donnell JS, Long GV, Scolyer RA, Teng MW, Smyth MJ. Resistance to PD1/PDL1 checkpoint inhibition. Cancer Treat Rev. (2017) 52:71-81. doi: 10.1016/j.ctrv.2016.11.007

65. Martins F, Sofiya L, Sykiotis GP, Lamine F, Maillard M, Fraga M, et al. Adverse effects of immune-checkpoint inhibitors: epidemiology, management and surveillance. Nat Rev Clin Oncol. (2019) 16:563-80. doi: 10.1038/s41571-019-0218-0

66. Swart M, Verbrugge I, Beltman JB. Combination approaches with immunecheckpoint blockade in cancer therapy. Front Oncol. (2016) 6:233. doi: 10.3389/fonc.2016.00233

67. Jin L, Lee EM, Ramshaw HS, Busfield SJ, Peoppl AG, Wilkinson L, et al. Monoclonal antibody-mediated targeting of CD123, IL-3 receptor alpha chain, eliminates human acute myeloid leukemic stem cells. Cell Stem Cell. (2009) 5:31-42. doi: 10.1016/j.stem.2009.04.018

68. Muñoz L, Nomdedéu JF, López O, Carnicer MJ, Bellido M, Aventín A, et al. Interleukin-3 receptor alpha chain (CD123) is widely expressed in hematologic malignancies. Haematologica. (2001) 86:1261-9.
69. Kobold S, Pantelyushin S, Rataj F, Vom Berg J. Rationale for combining bispecific $\mathrm{T}$ cell activating antibodies with checkpoint blockade for cancer therapy. Front Oncol. (2018) 8:285. doi: 10.3389/fonc.2018.00285

70. Krupka C, Kufer P, Kischel R, Zugmaier G, Lichtenegger FS, Köhnke $\mathrm{T}$, et al. Blockade of the PD-1/PD-L1 axis augments lysis of AML cells by the $\mathrm{CD} 33 / \mathrm{CD} 3$ BiTE antibody construct AMG 330: reversing a Tcell-induced immune escape mechanism. Leukemia. (2016) 30:484-91. doi: 10.1038/leu.2015.214

71. Waldmann TA. Cytokines in cancer immunotherapy. Cold Spring Harb Perspect Biol. (2018) 10:a028472. doi: 10.1101/cshperspect. a028472

72. Charych DH, Hoch U, Langowski JL, Lee SR, Addepalli MK, Kirk $\mathrm{PB}$, et al. NKTR-214, an engineered cytokine with biased IL2 receptor binding, increased tumor exposure, and marked efficacy in mouse tumor models. Clin Cancer Res. (2016) 22:680-90. doi: 10.1158/1078-0432.CCR15-1631

73. Siefker-Radtke AO, Baron AD, Necchi A, Plimack ER, Pal SK, Bedke J, et al. Nivolumab monotherapy in patients with advanced platinum-resistant urothelial carcinoma: efficacy and safety update from CheckMate 275. J Clin Oncol. (2019) 37:(Suppl. 15):4524. doi: 10.1200/JCO.2019.37.15_suppl.4524

74. Garris CS, Arlauckas SP, Kohler RH, Trefny MP, Garren S, Piot C, et al. Successful anti-PD-1 cancer immunotherapy requires $\mathrm{T}$ cell-dendritic cell crosstalk involving the cytokines IFN- $\gamma$ and IL-12. Immunity. (2018) 49:1148-61. doi: 10.1016/j.immuni.2018.09.024

75. Arora E, Masab M, Mittar P, Jindal V, Gupta S, Dourado C. Role of immune checkpoint inhibitors in advanced or recurrent endometrial cancer. Cureus. (2018) 10:e2521. doi: 10.7759/ cureus. 2521

Conflict of Interest: The authors declare that the research was conducted in the absence of any commercial or financial relationships that could be construed as a potential conflict of interest.

Copyright (๑) 2019 Marinelli, Annibali, Aguzzi, Tuyaerts, Amant, Morelli, Santoni, Amantini, Maggi and Nabissi. This is an open-access article distributed under the terms of the Creative Commons Attribution License (CC BY). The use, distribution or reproduction in other forums is permitted, provided the original author(s) and the copyright owner(s) are credited and that the original publication in this journal is cited, in accordance with accepted academic practice. No use, distribution or reproduction is permitted which does not comply with these terms. 